\title{
QUEEN'S
UNIVERSITY
BELFAST
}

\section{Predictors of nonadherence in children and adolescents with epilepsy: A multimethod assessment approach}

Alsous, M., Hamdan, I., Saleh, M., McElnay, J., Horne, R., \& Masri, A. (2018). Predictors of nonadherence in children and adolescents with epilepsy: A multimethod assessment approach. Epilepsy \& behavior : E\&B, 85, 205-211. https://doi.org/10.1016/j.yebeh.2018.06.022

Published in:

Epilepsy \& behavior : E\&B

Document Version:

Peer reviewed version

Queen's University Belfast - Research Portal:

Link to publication record in Queen's University Belfast Research Portal

Publisher rights

(C) 2018 Elsevier Inc. All rights reserved.

This manuscript version is made available under the CC-BY-NC-ND 4.0 license http://creativecommons.org/licenses/by-nc-nd/4.0/,which permits distribution and reproduction for noncommercial purposes, provided the author and source are cited

\section{General rights}

Copyright for the publications made accessible via the Queen's University Belfast Research Portal is retained by the author(s) and / or other copyright owners and it is a condition of accessing these publications that users recognise and abide by the legal requirements associated with these rights.

Take down policy

The Research Portal is Queen's institutional repository that provides access to Queen's research output. Every effort has been made to ensure that content in the Research Portal does not infringe any person's rights, or applicable UK laws. If you discover content in the Research Portal that you believe breaches copyright or violates any law, please contact openaccess@qub.ac.uk. 


\section{Predictors of non-adherence in children and adolescents with epilepsy: A multi-method assessment approach}

Mervat Alsous ${ }^{1}$, Imad Hamdan², Mohammad Saleh ${ }^{3}$, James McElnay ${ }^{4}$, Robert Horne ${ }^{5}$, Amira Masri6

${ }^{1}$ Associate Professor, Department of Clinical Pharmacy and Therapeutics, Faculty of Pharmacy, Applied Science Private University, Amman, Jordan.

${ }^{2}$ Professor, Department of Pharmaceutical Sciences, Faculty of Pharmacy, the University of Jordan, Amman, Jordan.

${ }^{3}$ Associate Professor, Department of Biopharmaceutics and Clinical Pharmacy, Faculty of Pharmacy, the University of Jordan, Amman, Jordan.

4Professor, Clinical and Practice Research Group, School of Pharmacy, Queen's University Belfast, Belfast, UK.

${ }^{5}$ Professor of Behavioural Medicine, Centre for Behavioural Medicine, UCL School of Pharmacy, University College London.

${ }^{6}$ Professor of children neurology, Faculty of medicine, The University of Jordan, Amman, Jordan. 


\title{
Predictors of non-adherence in children and adolescents with epilepsy: A multi-method assessment approach
}

\begin{abstract}
Background: There is a lack of a standardised tool for adherence measurement in patients with epilepsy. Studies in children with epilepsy have reported adherence in $50-96.5 \%$. The primary objective of this study was to identify predictors of non-adherence to Anti-Epileptic Drugs (AEDs) using two different methods in Jordanian children and adolescents with epilepsy.
\end{abstract}

Methods: Participants included 63 children and adolescents with epilepsy and their primary caregivers. Adherence measures included a subjective approach (using parent and child selfreports via Medication Adherence Report Scale (MARS) and an objective method (measuring plasma levels of AEDs coupled with the application of population pharmacokinetic models to predict AED concentrations in the children). The Beliefs about Medicines Questionnaire (BMQ) was used to examine the association beliefs about medicines with non-adherence in the participating patients.

Results: Measuring AEDs in plasma samples captured the highest percentage of nonadherence (36.2\%). No significant agreement was found between the AED plasma level method and both the MARS (parent) and MARS (child). The overall non-adherence (combined methods) to AED therapy in children with epilepsy was $44.4 \%$. Logistic regression analysis indicated that children with longer duration of disease were more likely (odds ratio [OR] 1.54, $95 \%$ confidence interval [CI] 1.16-2.04) to be classified as non-adherent as were children whose parents have lower AED Necessity scores (OR 0.68, 95\% CI $0.53-0.87$ ) and higher AED Concerns (OR 1.6, 95\% CI 1.26-2.04) as measured by the BMQ.

\section{Conclusion:}

The use of a multi-method approach for assessing adherence increases sensitivity for detection of non-adherence to AEDs. Disease duration and parental Necessity beliefs and Concerns assessed by the BMQ-Specific questionnaire were significant predictors of nonadherence to the AED therapy. The need for the development and implementation of interventions that can be employed to improve adherence within this paediatric population has been highlighted by the high levels of non-adherence identified.

Keywords: Anti-Epileptic Drugs, adherence, Medication Adherence Report Scale (MARS), Children, BMQ, Necessity Concerns Framework 


\section{Predictors of non-adherence in children and adolescents with epilepsy: A multi-method assessment approach}

\section{Introduction}

Worldwide, 50 million people have epilepsy [1] and 10.5 million of them are children under 15 years old [2]. Adherence assessment and factors that affect adherence in children with epilepsy are not widely researched [3]. The term of medication adherence can be defined as "the extent to which patients take medication as prescribed by their health care providers" [4].Successful therapy using anti-epileptic drugs (AEDs) can eliminate or reduce symptoms; adherence to AEDs is subsequently a key to treatment success [5].

Reports on the extent of non-adherence to (AEDs) in patients with epilepsy vary considerably, ranging from $3.5 \%$ to $68 \%$ in children $[3,6-7]$ and from $28.9-74.8 \%$ in adults [8-11] depending on the population studied and method used to assess adherence.

Generally there are several factors that can influence medication adherence to AEDs in adolescents including regimen complexity or their beliefs about medication as they may stop taking the medication because they do not want to be viewed as being different than others [12]. Research on children population has revealed that disliking the taste of medication, running out of medications [13], parent forgetfulness, refusal to take medications $[13,14]$, difficulties in swallowing the medication [14], lower socioeconomic status [15-17] and family conflict and difficulties in communication particularly during adolescence [17] were barriers to adherence to AEDS. Poor adherence to prescribed AEDs is associated with negative consequences such as reduced seizure control [18-20], higher incidence of hospital admissions [20-22] and 
increased health care costs $[5,23,24]$. Adherence assessment in children newly diagnosed with epilepsy is critical for clinicians to make accurate treatment decisions such as changing the dose of the drug [25]. In addition, clinicians should take into consideration that White coat adherence may occur at the beginning of the treatment and therefore it should be examined to avoid unnecessary changes to medication treatment [26].

Early adherence interventions could change the seizure control, particularly if variability in adherence was minimized primarily after diagnosis [27-28]. Examples of such interventions include text messaging and application-based interventions to increase adolescent adherence to AEDs [29], supporting treatment adherence regimens [30] and family-tailored adherence intervention targeting non-adherence to AEDs in children with new-onset epilepsy [31].

There is no standardised tool for adherence measurement in patients with epilepsy [32]. Several studies have used the Morisky scale [33] or the Medication Adherence Report Scale (MARS) scale[3, 34] as self-report measures to assess adherence in patients with epilepsy[18, 35] and the Pediatric Epilepsy Medication SelfManagement Questionnaire which have been validated in adolescents [36] and caregivers of children [37]. Other researchers have assessed adherence using 'pill' counts [38] electronic monitors [6, 39,40] and medication refills [5, 23, 41, 42].

Adherence assessment using objective methods such as blood level determination of AEDs has also been utilised in various studies [3, 43-47].

Each adherence assessment method has its advantages and disadvantages [4]. In general, objective methods are more accurate in measuring adherence, however, this approach is expensive and associated with difficulties in terms of developing assays 
and interpretation of results [4]. Moreover, variability in the pharmacokinetics of the AEDs due to variable absorption, drug interactions with medications taken concomitantly and differences in rates of drug metabolism may influence the results obtained [45]. Utilizing established PopPK models of the AEDs for the prediction of concentration of the AED by replacing the covariates in the models with patients' actual values (i.e. time of sampling, age, dose, body weight) minimizes the intraand inter-individual variability associated with the measured concentrations and provide a good approach to accurately assess the blood level of AED [3].

For drugs with short half-lives, taking doses in some cases a few hours before a test raises the drug level reasonably close to target, thus depending on drug concentration measurement as a single method for assessing adherence can be misleading.

Subjective methods which include asking patients questions about their medication taking behaviour (questionnaires) are less accurate measures of adherence, however, they are more convenient and usually less expensive [4].

Adherence is best determined by using different assessment approaches and triangulating the results obtained to provide an overall assessment [45].

Patients' beliefs about their prescribed medicines (BMQ), involves assessment of patients' beliefs about medication prescribed for a particular illness. It also has two major themes, which are 'Necessity Beliefs' and 'Concerns'. Patients' views about the necessity of the medication for maintaining or improving health describe the 'Necessity Beliefs' whereas beliefs about the potential adverse effects of taking medication explain the 'Concerns' [48]. This 'Necessity-Concerns Framework (NCF)' possibly is considered as a useful model for health care providers to identify and 
address key beliefs supporting patients' attitudes and judgments about treatment [49].

The main aim of the present study was to use subjective and objective approaches to assess the level of adherence to prescribed AEDs in children with epilepsy. Additional aim was to identify factors that influence adherence including patients' beliefs about AEDs. It was hypothesised that low scores of the BMQ-necessity subscale and high scores of the BMQ-concern subscale administered to parents would be associated with poor medication adherence in the recruited children.

\section{Methods}

\section{Study design}

This cross sectional study was carried out at the child neurology clinic at Jordan University Hospital (JUH), a tertiary care hospital in Amman, the capital of Jordan. The study was approved by the Research Ethics Committee of the University of Jordan Hospital. The study was carried out between October 2015 and November 2016. Subjective adherence to AEDs was assessed in children with epilepsy using a validated Arabic version of the Medication Adherence Report Scale (MARS) [50] and by measuring AED plasma levels (objective method).

Factors affecting adherence to antiepileptic drugs were determined by using Parent necessity and concern subscales of the validated Arabic version of the Beliefs about Medicines Questionnaire (BMQ)-specific [50].

The overall seizure severity of each participating child was assessed by their physician, using the Global Assessment of Severity of Epilepsy (GASE) Scale [51]. 


\section{Study patients and data collection}

Children with epilepsy aged $\leq 18$ years and who were receiving AEDs [including one or more of the following: carbamazepine (CBZ), levetiracetam (LVT), sodium valproate (VPA)] for at least one month were included. After screening of the prescribed medications in the hospital to children with Epilepsy it was noted that those 3 drugs were the most prescribed medications. The inclusion of all antiepileptic drugs would be better however, due to financial limitations these 3 drugs were chosen in order to recruit higher percentage of patients.

Parents were asked to sign a consent form after receiving a full explanation of the study and only children whose parents provided written informed consent were included. Assent was also obtained from children who were considered capable of providing this by their physician.

The medical files were reviewed to collect data on patients ' demographics, current medications and medical history (changes to medications, type of seizures and disease duration).

\section{Study measures and adherence assessment}

The MARS and BMQ-Specific questionnaires were self-completed by parents and their children (if $\geq 11$ years old, during the clinic visit). Capability of children to complete the questionnaire was determined by his/her physician.

\section{Medication Adherence Report Scale (MARS)}

The validated Arabic translations of the MARS questionnaires (parent and child version) were used [50] to assess adherence to medication in the past month. The child's version consists of five items; mean scores were summed to give a scale score ranging from 1 to 5 . The parent's version consists of six questions; mean scores were 
summed to give a scale score ranging from 1 to 5 . The parent MARS questionnaire is the same as the patient/child questionnaire, except for the last item that is not relevant to the child, i.e. "I don't give it because my child refuses it". Higher scores indicate higher levels of self-reported adherence. In the present study, a $90 \%$ cutoff point for adherence was used, i.e. a participant was considered to be adherent, if the parental/child MARS score was at least 4.5 out of 5 . This cut off-point was used in a previously published studies for the assessment of adherence to medication in children $[3,52]$

\section{AED concentration in plasma samples}

Blood samples $(2 \mathrm{ml}$ ) were collected from each participating child at a clinic visit (the date that the participants completed the study questionnaires). All samples were labelled with patient study number, the date and time of collection. Plasma was obtained for each sample after blood centrifugation and stored at $-80^{\circ} \mathrm{C}$ until AED analysis was performed. For VPA and CBZ measurement was performed using (chemiluminescent microparticle immunoassay (CIMA). For measurement of LVT, a validated micro-analytical method using high-performance liquid chromatography (HPLC) with UV detection (210 nm) was utilized [53]. The concentrations of AEDs were expressed in $\mu \mathrm{g} / \mathrm{ml}$.

To determine whether a child was adherent, a pharmacokinetic simulation method was utilized to estimate the 0.95 interval of predicted plasma concentrations for each AED at the time of sampling in individual patients $(n=1,000$ sets of simulations using the non-linear mixed effect modeling software package, NONMEM, Icon Development Solutions, Ellicott City, MD, U.S.A.). In this methodology, literature 
values of population pharmacokinetics (PopPK) parameters for each drug were employed [54-56]. In addition, significant covariates reported to influence PK parameters for each drug (e.g. age, dose, body weight) were incorporated into the simulation models.

Patients were considered adherent if their measured AED concentrations were within the calculated $95 \%$ prediction intervals. Patients taking more than one drug of interest were categorized as non-adherent if the measured concentration was lower or higher than the simulated concentration for at least one of the AEDs prescribed.

\section{Overall Adherence assessment}

Adherence to AEDs was assessed using subjective (MARS) and objective (plasma concentrations) methods:

a) If score is $\geq 4.5$ using the parent/guardian or child MARS questionnaire then patient was deemed adherent.

b) Level of AED was within the calculated $95 \%$ prediction intervals. Patients taking more than one AED were categorized as non-adherent if the measured concentration was lower or higher than the simulated concentration for at least one of the AEDs prescribed. Patients taking more than one AED of interest were categorized as nonadherent if the measured concentration was lower or higher than the simulated concentration for at least one of the AEDs prescribed.

Finally, using all data available, a patient was classified as non-adherent if one of the adherence measures was indicative of non-adherence. 


\section{Beliefs about Medicines Questionnaire (BMQ-Specific)}

The validated Arabic translation of the BMQ-Specific questionnaires (parent and child versions) were used [50]. The BMQ-Specific involves assessment of patients' beliefs about medication prescribed for a specific illness, in this case AED. It comprises two scales: the AED-Necessity scale comprises 5 items assessing perceptions of personal need for AED to maintain or improve health now and in the future. The AED-Concerns scale comprises 6 items assessing concerns about AED. For each scale, Adjusted Scale Mean Scores were computed by summing the individual item scores and dividing by the number of items in the scale (range 1-5). Higher scores indicate stronger perceptions of the constructs represented by the scales.

\section{Data analysis}

All analyses were carried out using SPSS version 22 (SPSS Inc., Chicago, IL, U.S.A.). Group differences (adherent vs. non-adherent) were explored using MannWhitney $U$ analysis for continuous variables and using the chi-square or Fisher's exact test for categorical variables. The significance level was set at 0.05 throughout analyses. The magnitude of agreement between different adherence assessment methods was determined using the Kappa ( $\mathrm{K}$ ) coefficient [57].

Screening of the initial datasets was performed using univariate analysis (including demographic data, disease characteristics, overall epilepsy severity using GASE scale beliefs about medications, type of AED of interest prescribed, number of AEDs prescribed, total number of medications and parent education). According to univariate analysis, three significant factors were shown to have significant effect on non-adherence $(p<0.05)$, (i) the disease duration, (ii) Parent BMQ-Necessity score 
and (iii) Parent BMQ-concern score. These significant variables were subjected to multivariate analysis using binary logistic regression to evaluate the potential predictors of non-adherence to AEDs.

\section{Results}

\section{Patients demographic Characteristics}

The parents of eighty seven eligible patients were approached and sixty three agreed to take part in this cross sectional study. Four participating children refused to give blood. About half of the patients received LVT. The demographics and disease characteristics of the study subjects are described in Table 1 . The number of medications received by patients ranged between 1-6 medications (median $=1$ ).

\section{Adherence assessment}

\section{Adherence using MARS specific (parent and child versions)}

Score distribution for the MARS questionnaires is presented in Table 2. Children were classified as being adherent if they recorded a total score $\geq$ the $90^{\text {th }}$ percentile of the maximum score [i.e $\geq 4.5$ for MARS (parents and child)]. Accordingly, using the parental MARS questionnaire scores, a total of 50 children $(79.4 \%)$ were classified as being adherent. On the other hand, among the 21 children who answered the MARS (child) questionnaire (those aged 11 years or above), fourteen children $(66.7 \%)$ were classified as being adherent.

The mean MARS score for parents [4.67, SD (0.5)] was higher than mean MARS score for children [4.34, SD (0.17); $p=0.042]$.

\section{AED concentrations in plasma samples}


A total of 58 plasma samples were obtained from the 63 patients recruited in the study i.e. four patients refused to give a blood sample for analysis and one blood sample was of insufficient volume for analysis. Sixteen patients (27.6\%) had AED plasma concentrations lower than the $95^{\text {th }}$ percentile of the predicted concentrations according to the PopPK modeling while five patients were found to be over adherent $(8.6 \%)$. Twenty one patients were therefore deemed non-adherent using this measure (36.2\%). Measured AED concentrations (median and range) are shown in Table 1.

\section{Assessment of different measures of adherence}

Measuring AEDs in plasma samples captured the highest percentage of nonadherence (36.2\%) followed by the MARS (child) questionnaire (33.3\%). The lowest percentage of non-adherence $(20.6 \%)$ was observed using the MARS (parent) questionnaire, Figure. 1. When all the three methods were taken into consideration $44.4 \%$ of children were classified as non-adherent.

Only 7 patients $(12.07 \%)$ of patients were deemed non-adherent using (subjective and objective methods) while 14 patients (24.1) were considered non-adherent using objective methods and adherent according to subjective methods

The interrater reliability agreements across the different adherence assessment methods are presented in Table 3. The interrater reliability for the MARS (parent) and MARS (child) was found to be significant (Kappa $=0.64$, p-value $=0.002$ ) indicating a significant agreement between the two assessment methods. On the other hand, no significant agreement was found between the AED plasma level method and both the MARS (parent) and MARS (child). 


\section{Beliefs about medicines}

Sixty three parents and twenty one children completed the BMQ-specific questionnaire. The majority of parents (88.9\%) and children $(76.2 \%)$ had a strong belief about the necessity of AEDs (BMQ-specific scores above scale midpoint), whereas approximately $76.2 \%$ (parents) and $61.9 \%$ (children) had concerns about AED harmful effects. The median total scores for the necessity and concern subscales were 3.8 (range 2-5) and $3.2(2-5)$ for parents and $3.8(1.0-4.6)$ and $3.0(2.2-4.2)$ for children, respectively. Reliability coefficients (Chronbach's alpha) for the necessity and concern subscales were 0.707 and 0.769 for parents and 0.802 and 0.747 for children respectively, indicating reliability of the methodology.

\section{Factors affecting adherence to antiepileptic drugs}

Univariate analysis showed that parental beliefs about their child's AED (BMQNecessity and BMQ-Concerns) and disease duration were associated with overall adherence to AEDs. These variables were subjected to logistic regression analysis (backward LR). The analysis indicated that increased disease duration and higher scores for the BMQ-Concerns (parent) scale and lower scores for the parental BMQNecessity scale were significantly and independently associated with non-adherence Table 4. The mean disease duration in adherent patients [3.10, SD (0.54) was shorter than in those who were deemed non-adherent [5.56, SD (0.71)], p-value=0.002. The mean BMQ Necessity score (parent) in adherent patients [19.63, SD (3.08) was higher than in those who were deemed non-adherent [17.17, SD (3.50)], pvalue $=0.004$. The mean BMQ-Concern score (parent) in adherent patients [17.29, 
SD (4.27] was lower than those who were deemed non-adherent [21.36, SD (3.75)], p-value $<0.001$

\section{Discussion}

This is the first study in Jordan that investigated the adherence to AEDs in children and adolescents utilizing a combined subjective method (self-reported questionnaires) and an objective method (measuring AEDs in plasma samples) to better describe non-adherence to anti-epileptic treatment.

Adherence to AED therapy is crucial for effective disease management, yet nonadherence rates are high due to several barriers [58]. The adherence rate varies depending on the unique characteristics of the population being studied (e.g. differences in the patients' attitude toward adherence due to cultural or educational influences, or their clinicians' approach and method being used to assess adherence[35, 59].

In the present study, there was a significant agreement between child-reported and parent-reported adherence rates $($ Kappa $=0.64, \mathrm{p}$-value $=0.002)$, and this follows the trend of good parent-child agreement from prior studies $[3,60]$. Parent-reported adherence rates $(79.4 \%)$ were, however, greater than child-reported adherence rates $(66.7 \%)$ highlighting the subjectivity of the self-reported approach, i.e. perception of adherence using self-reported approach may vary by reporter. Lower self-reported adherence rates by children and adolescents are perhaps due to increased independence in taking the medication, while parents are more likely to present their child as adhering to prescribed regimens and not admit to going against doctors' advice. 
The highest percentage of non-adherence was observed with plasma AED concentrations (36.2\%). This reflects the known overestimation of adherence using subjective methods[6,61]. In addition, no significant agreement was found between the AED plasma level method and both the MARS (parent) and MARS (child). It is possible that AEDs plasma levels were affected by the behavior of the patient in the days immediately preceding the blood draws. A patient could miss doses before obtaining blood sample and be identified as poorly adherent by the levels of AEDs in the plasma, even though over a month period the individual had taken regularly his or her medication.

The application of PopPK models, to predict AED concentrations in individual children according to the relevant covariates present (i.e. time of sampling, age, dose, body weight) minimizes the intra- and inter-individual variability associated with the measured concentrations and this approach has been shown to be useful for estimating levels of adherence in children with epilepsy [3]. Incorporation of PopPK models into clinical practice to assess adherence for each patient has a potential benefit for clinicians to make accurate treatment decisions.

The overall non-adherence rate to the AEDs when the results of different methods were combined was $44.4 \%$, which was considered high, however, it is within the reported range in published literature on the non-adherence to treatment in children with epilepsy of $3.5-58 \%[3,6,35,39]$. The use of a multi-method approach increases the sensitivity of detecting non-adherence to treatment in patients $[3,62]$. It was possible that non-adherence was overestimated in the present study, as patients were classified as being non-adherent if one of the assessment methods employed was indicative of non-adherence, despite other measures implying 
adherence. Furthermore, five patients were classified as over-adherent using the plasma analysis. Over-adherence more often occurs in patient with complex drug regimen. It is considered a significant clinical problem as and may lead to increased side effects of treatment [63]. The small sample size and the diversity within the study population in regards to regimen complexity and patient's behavior toward taking the medication may have influenced the findings. The current research could be considered as a preparatory work for a national study in this field, with a larger population of children.

Patients' demographic data (i.e. age, gender and number of prescribed medicines) were not found to be predictors of non-adherence in the present study. This was consistent with previous studies in the literature $[39,64,65]$. Longer disease duration was significantly and independently associated with non-adherence. This finding is similar to the results reported by previous studies $[66,67]$.

Studies linking parental beliefs about necessity of drugs in their children and adherence is limited [3]. A study by Shah et al [3] in Northern Ireland reported that that parents' beliefs toward a greater need for AEDs in managing epilepsy was a barrier to medication adherence to AEDS. In the present study, children of parents with negative beliefs about their child's medicine were more likely to be classified as non-adherent to AEDs. This can be due to clinic setting in JUH and time constraint which are challenging factors to an effective patient counselling about prescription necessity and benefit to patient. Our findings confirm the applicability of the Necessity-Concerns Framework [68] and results are consistent with previous adult studies linking non-adherence to AEDs to patients' beliefs about AEDs[69-71]. The present results have implications for the provision of adherence support to parents 
and children. They suggest the need to apply a Perceptions and Practicalities Approach to supporting informed adherence [72] as recommended by the NICE Medicines Adherence Guidelines [73] This emphasises the need to address not just the practicalities of adherence (eg ability to use the medication as advised) but also the perceptions that influence motivations to adhere. Consistent with NICE our findings suggest the importance of the Necessity Concerns Framework in identifying salient adherence-related perceptions of AED. Adherence support should be tailored to communicate a common-sense rationale for the necessity of regular AED and to elicit and address specific concerns about AED. Epilepsy nurses or clinical pharmacists, may be suitable as delivery channel for this type of adherence to parents and children but further studies are needed to demonstrate the efficacy of this approach. Our finding suggests that efforts of education and counselling by health care provider should target parents to reduce misconceptions and wrong beliefs about AEDs use. Further evaluation of reasons for non-adherence to AEDs in children in Jordan using prospective longitudinal designs should also be undertaken to support the development of appropriate adherence interventions for implementation into routine paediatric clinical practice. These educational and integrated care interventions should improve insight and understanding of patients' perceptions and beliefs about using AEDs.

\section{Limitations}

The findings of this study should be considered within the context of a few limitations. Firstly, a modest sample size was used, within which there was a narrow range of demographic characteristics. Future work should include a larger sample size 
consisting of a more even proportion of patients within gender, diagnosis, disease severity, family socioeconomic status and medication type categories. Secondly, the study design was observational, cross-sectional and therefore could not assess cause-effect relationship between parental beliefs and adherence. This can be further assessed using prospective longitudinal designs. A further potential limitation of the study is that only one sample was obtained per patient which might reflect shortterm adherence more than long term adherence. Multitude factors that may affect serum levels, such as missing one or two doses in the past weeks versus missing several doses over the course of the month, should be addressed in future studies by the inclusion of multiple samples withdrawn at various time points. On the other hand, this highlights the importance of using multi-method approach for accurate assessment of adherence.

\section{Conclusions}

The overall adherence to AED therapy in children in Jordan with epilepsy was $55.6 \%$, which was within the range reported in the literature. Adherence assessment using multi-method approach, via self-reported questionnaires and measurement of the AED concentrations coupled with the use of published PopPK models for predicting AED plasma concentrations, was shown to be a valuable approach for estimating levels of adherence. Logistic regression analyses identified the disease duration and parental AED Necessity and AED concern beliefs assessed by the BMQ-specific questionnaire as significant predictors of non-adherence to the AED therapy. There is a need for the development and implementation of clinical interventions aimed at overcoming the high level of non-adherence in children with epilepsy i.e. strives to increase adherence to more acceptable levels. These interventions by health care 
providers should actively involve patients and their parents by reinforcement of learned information, and positive confirmation of behavior changes and counselling about prescribed drug to reduce misconception about the use of medication.

\section{Acknowledgments}

The author would like to thank the patients and their families who participated in the study. The authors are grateful to the Applied Science Private University, for the financial support granted to perform this research project (Grant No. 2013-2014/39).

\section{Conflict of interest}

The authors declare no conflict of interest. 


\section{References}

1. Leonardi, M. and T.B. Ustun, The global burden of epilepsy. Epilepsia, 2002. 43(s6): p. 21-25.

2. Guerrini, R., Epilepsy in children. The Lancet, 2006. 367(9509): p. 499524.

3. Shah, N.M., et al., Adherence to antiepileptic medicines in children: A multiple-methods assessment involving dried blood spot sampling. Epilepsia, 2013. 54(6): p. 1020-1027.

4. Osterberg, L. and T. Blaschke, Adherence to medication. N Engl J Med, 2005. 353(5): p. 487-497.

5. Jacobs, K., et al., Medicine possession ratio as proxy for adherence to antiepileptic drugs: prevalence, associations, and cost implications. Patient Prefer Adherence, 2016. 10: p. 539.

6. Modi, A.C., et al., Development and reliability of a correction factor for parent-reported adherence to pediatric antiepileptic drug therapy. Epilepsia, 2011. 52(2): p. 370-376.

7. Hodges, J.C., et al., Identification and prevention of antiepileptic drug noncompliance: the collaborative use of state-supplied pharmaceutical data. ISRN pediatrics, 2014.

8. Zeber, J.E., L.A. Copeland, and M.J.V. Pugh, Variation in antiepileptic drug adherence among older patients with new-onset epilepsy. Ann Pharmacother, 2010. 44(12): p. 1896-1904.

9. Chen, H.-F., et al., The relationships among medicine symptom distress, self-efficacy, patient-provider relationship, and medication compliance in patients with epilepsy. Epilepsy Behav, 2010. 19(1): p. 43-49.

10. Sweileh, W.M., et al., Self-reported medication adherence and treatment satisfaction in patients with epilepsy. Epilepsy Behav, 2011. 21(3): p. 301-305.

11. Chinnaiyan S., Narayana S., Nanjappa V.P. Adherence to antiepileptic therapy in adults. Journal of neurosciences in rural practice, 2017. 8(3): 417.

12. Garnett, W.R., Antiepileptic drug treatment: outcomes and adherence. Pharmacotherapy: The Journal of Human Pharmacology and Drug Therapy, 2000. 20(8P2): p. 191S-199S.

13. Gutierrez-Colina, A. M., Smith, A. W., Mara, C. A. and Modi, A. C, Adherence barriers in pediatric epilepsy: From toddlers to young adults. Epilepsy \& Behavior, 2018. 80, 229-234.

14. Ramsey, R. R., Zhang, N., \& Modi, A. C, The Stability and Influence of Barriers to Medication Adherence on Seizure Outcomes and Adherence in Children With Epilepsy Over 2 Years. Journal of pediatric psychology, 2017. 
15. Modi, A. C., Rausch, J. R., \& Glauser, T. A, Patterns of nonadherence to antiepileptic drug therapy in children with newly diagnosed epilepsy. Jama, 2011. 305(16), 1669-1676.

16. Loiselle, K., Rausch, J. R., \& Modi, A. C, Behavioral predictors of medication adherence trajectories among youth with newly diagnosed epilepsy. Epilepsy \& Behavior, 2015. 50, 103-107.

17. Smith, A. W., Mara, C. A., \& Modi, A. C, Adherence to antiepileptic drugs in adolescents with epilepsy. Epilepsy \& Behavior, 2018. 80, 307311.18. Cramer, J.A., M. Glassman, and V. Rienzi. The relationship between poor medication compliance and seizures. Epilepsy Behav, 2002. 3(4): p. 338-342.

19. Jones, R., et al., Adherence to treatment in patients with epilepsy: associations with seizure control and illness beliefs. Seizure, 2006. 15(7): p. 504-508.

20. Hovinga, C.A., et al., Association of non-adherence to antiepileptic drugs and seizures, quality of life, and productivity: survey of patients with epilepsy and physicians. Epilepsy Behav, 2008. 13(2): p. 316-322.

21. Faught, E., et al., Nonadherence to antiepileptic drugs and increased mortality Findings from the RANSOM Study. Neurology, 2008. 71(20): p. $1572-1578$.

22. Samsonsen, C., et al., Nonadherence to treatment causing acute hospitalizations in people with epilepsy: an observational, prospective study. Epilepsia, 2014. 55(11): p. e125-e128.

23. Davis, K.L., S.D. Candrilli, and H.M. Edin, Prevalence and cost of nonadherence with antiepileptic drugs in an adult managed care population. Epilepsia, 2008. 49(3): p. 446-454.

24. Faught, R.E., et al., Impact of nonadherence to antiepileptic drugs on health care utilization and costs: findings from the RANSOM study. Epilepsia, 2009. 50(3): p. 501-509.

25. Modi, A. C., Wu, Y. P., Guilfoyle, S. M., \& Glauser, T. A, Uninformed clinical decisions resulting from lack of adherence assessment in children with new-onset epilepsy. Epilepsy \& Behavior, 2012. 25(4), 481-484.

26. Modi, A. C., Ingerski, L. M., Rausch, J. R., Glauser, T. A., \& Drotar, D, White coat adherence over the first year of therapy in pediatric epilepsy. The Journal of pediatrics, 2012. 161(4), 695-699.

27. Modi, A. C., Rausch, J. R., \& Glauser, T. A, Early pediatric antiepileptic drug nonadherence is related to lower long-term seizure freedom. Neurology, 2014. 82(8), 671-673.

28. Modi, A. C., Wu, Y. P., Rausch, J. R., Peugh, J. L., \& Glauser, T. A, Antiepileptic drug nonadherence predicts pediatric epilepsy seizure outcomes. Neurology, 2014. 83(22), 2085-2090.

29. Modi, A. C., Mann, K. A., Urso, L., \& Peugh, J, Preliminary feasibility and efficacy of text messaging and application-based adherence 
interventions in adolescents with epilepsy. Epilepsy \& Behavior, 2016. 63, 46-49.

30. Modi, A. C., Guilfoyle, S. M., Mann, K. A., \& Rausch, J. R, A pilot randomized controlled clinical trial to improve antiepileptic drug adherence in young children with epilepsy. Epilepsia, 2016. 57(3).

31. Modi, A. C., Guilfoyle, S. M., \& Rausch, J, Preliminary feasibility, acceptability, and efficacy of an innovative adherence intervention for children with newly diagnosed epilepsy. Journal of pediatric psychology, 2013. 38(6), 605-616.

32. Paschal, A.M., et al., Measures of adherence to epilepsy treatment: review of present practices and recommendations for future directions. Epilepsia, 2008. 49(7): p. 1115-1122.

33. Morisky, D.E., L.W. Green, and D.M. Levine, Concurrent and predictive validity of a self-reported measure of medication adherence. Medical care, 1986. 24(1): p. 67-74.

34. Chapman, S.C., et al., Applying a perceptions and practicalities approach to understanding nonadherence to antiepileptic drugs. Epilepsia, 2015. 56(9): p. 1398-1407.

35. McAuley, J.W., et al., An evaluation of self-management behaviors and medication adherence in patients with epilepsy. Epilepsy Behav, 2008. 13(4): p. 637-641.

36. Carbone, L., Zebrack, B., Plegue, M., Joshi, S., \& Shellhaas, R, Treatment adherence among adolescents with epilepsy: what really matters?. Epilepsy \& Behavior, 2013. 27(1), 59-63.

37. Modi, A. C., Monahan, S., Daniels, D., \& Glauser, T. A, Development and validation of the pediatric epilepsy medication self-management questionnaire. Epilepsy \& Behavior, 2010. 18(1), 94-99.

38. Lisk, D. and S. Greene, Drug compliance and seizure control in epileptic children. Postgrad Med J, 1985. 61(715): p. 401-405.

39. Modi, A.C., D.A. Morita, and T.A. Glauser, One-month adherence in children with new-onset epilepsy: white-coat compliance does not occur. Pediatrics, 2008. 121(4): p. e961-e966.

40. Ingerski, L. M., Hente, E. A., Modi, A. C. and Hommel, K. A, Electronic measurement of medication adherence in pediatric chronic illness: a review of measures. The J ournal of pediatrics, 2011. 159(4), 528-534.

41. Briesacher, B.A., et al., Comparison of drug adherence rates among patients with seven different medical conditions. Pharmacotherapy, 2008. 28(4): p. 437-443.

42. Gollwitzer, S., et al., Nonadherence to antiepileptic drugs in Germany A retrospective, population-based study. Neurology, 2016. 87(5): p. 466472.

43. Hazzard, A., S.J. Hutchinson, and N. Krawiecki, Factors related to adherence to medication regimens in pediatric seizure patients. J Pediatr Psychol, 1990. 15(4): p. 543-555. 
44. Gomes, M.d.M. and H.d.S. Maia Filho, Medication-taking behavior and drug self regulation in people with epilepsy. Arq Neuropsiquiatr, 1998. 56(4): p. 714-719.

45. Mitchell, W.G., L.M. Scheier, and S.A. Baker, Adherence to treatment in children with epilepsy: who follows" doctor's orders"? Epilepsia, 2000. 41(12): p. 1616-1625.

46. Specht, U., et al., Postictal serum levels of antiepileptic drugs for detection of noncompliance. Epilepsy Behav, 2003. 4(5): p. 487-495.

47. Kemp, S., et al., Psychological factors and use of antiepileptic drugs: pilot work using an objective measure of adherence. Psychology, Health and Medicine, 2007. 12(1): p. 107-113.

48. Horne R., Weinman J. Patients' beliefs about prescribed medicines and their role in adherence to treatment in chronic physical illness. Journal of psychosomatic research, 1999. 47(6):555-67.

49. Horne R., Chapman S.C., Parham R., Freemantle N., Forbes A., Cooper $\mathrm{V}$. Understanding patients' adherence-related beliefs about medicines prescribed for long-term conditions: a meta-analytic review of the Necessity-Concerns Framework. PloS one, 2013. 8(12):e80633.

50. Alsous M, Alhalaiqa F, Abu Farha R, Abdel Jalil M, McElnay J, Horne R. Reliability and Validity of Arabic Translation of Medication Adherence Report Scale (MARS) and Beliefs about Medication Questionnaire (BMQ) - Specific for Use in Children and their Parents. PLOS one. 2017.

51. Speechley, K.N., et al., Assessing severity of epilepsy in children: preliminary evidence of validity and reliability of a single-item scale. Epilepsy Behav, 2008. 13(2): p. 337-342.

52. Alsous, M., Farha, R.A., Alefishat, E., Al Omar, S., Momani, D., Gharabli, A., McElnay, J., Horne, R and Rihani, R. (2017). Adherence to 6Mercaptopurine in children and adolescents with Acute Lymphoblastic Leukemia. PloS one, 12(9), e0183119.

53. Pucci, V., et al., High-performance liquid chromatographic determination of Levetiracetam in human plasma: comparison of different sample clean up procedures. Biomed Chromatogr, 2004. 18(1): p. 37-44.

54. Yukawa, E., et al., Population-Based Investigation of Valproic Acid Relative Clearance Using Nonlinear Mixed Effects Modeling: Influence of Drug-Drug Interaction and Patient Characteristics. Journal of Clinical Pharmacology, 1997. 37(12): p. 1160-1167.

55. Reith, D.M., et al., Population pharmacokinetic modeling of steady state carbamazepine clearance in children, adolescents, and adults. J Pharmacokinet Pharmacodyn, 2001. 28(1): p. 79-92.

56. Chhun, S., et al., Population pharmacokinetics of levetiracetam and dosing recommendation in children with epilepsy. Epilepsia, 2009. 50(5): p. 1150-1157. 
57. Landis, J.R. and G.G. Koch, The measurement of observer agreement for categorical data. biometrics, 1977: p. 159-174.

58. Faught, E., Adherence to antiepilepsy drug therapy. Epilepsy Behav, 2012. 25(3): p. 297-302.

59. Malik, M.A., et al., Medication Nonadherence in Children with Epilepsy Attending Outpatient Clinics in Under-Resourced Community. J Pediatr Epilepsy, 2015. 4(02): p. 072-079.

60. Dolezal, C., et al., The reliability of reports of medical adherence from children with HIV and their adult caregivers. J Pediatr Psychol, 2003. 28(5): p. 355-361.

61. Daniels, T., et al., Accurate assessment of adherence: self-report and clinician report vs electronic monitoring of nebulizers. Chest, 2011. 140(2): p. 425-432.

62. Goodfellow, N.A., et al., Adherence to treatment in children and adolescents with cystic fibrosis: a cross-sectional, multi-method study investigating the influence of beliefs about treatment and parental depressive symptoms. BMC Pulm Med, 2015. 15(1): p. 1.

63. You M., Veronica Decker MS R.N. Issues related to overadherence to oral chemotherapy or targeted agents. Clinical journal of oncology nursing, 2013. 17(6):604.

64. Liu, J., et al., Adherence to treatment and influencing factors in a sample of Chinese epilepsy patients. Epileptic Disord, 2013. 15(3): p. 289-294.

65. Ogboi Sonny, J., et al., Evaluation of factors influencing medication adherence in patients with epilepsy in rural communities of Kaduna State, Nigeria. Neuroscience \& Medicine, 2011. 2011.

66. Mbuba, C.K., et al., Risk factors associated with the epilepsy treatment gap in Kilifi, Kenya: a cross-sectional study. Lancet Neurol, 2012. 11(8): p. 688-696.

67. Al Jumah K., Hassali M.A., Al Qhatani D., El Tahir K. Factors associated with adherence to medication among depressed patients from saudi arabia: a cross-sectional study. Neuropsychiatric disease and treatment, 2014. 10:2031.

68. Horne, R., et al. Doubts about necessity and concerns about adverse effects: identifying the types of beliefs that are associated with nonadherence to HAART. International Journal of STD and AIDS, 2004. 15(1): p. 38-44.

69. Chapman, S., et al., Patients' perspectives on antiepileptic medication: relationships between beliefs about medicines and adherence among patients with epilepsy in UK primary care. Epilepsy Behav, 2014. 31: p. 312-320.

70. Nakhutina, L., et al., Adherence to antiepileptic drugs and beliefs about medication among predominantly ethnic minority patients with epilepsy. Epilepsy Behav, 2011. 22(3): p. 584-586. 
71. Bautista, R.E.D., C. Graham, and S. Mukardamwala, Health disparities in medication adherence between African-Americans and Caucasians with epilepsy. Epilepsy Behav, 2011. 22(3): p. 495-498.

72. Horne R. Compliance, adherence, and concordance: implications for asthma treatment. Chest 2006;130(1 Suppl):65S-72S

73. NICE. Clinical Guideline 76: Medicines adherence: Involving patients in decisions about prescribed medicines and supporting adherence, 2009. 
Table 1. Demographic and disease characteristics of the study sample $(n=63)$

\begin{tabular}{|c|c|}
\hline Children age (years), mean (SD) & $8.36(4.22)$ \\
\hline Range (Years) & $1.5-18$ \\
\hline Children weight (kg), mean (SD) & $29.09(14.30)$ \\
\hline \multicolumn{2}{|l|}{ Children gender, n (\%) } \\
\hline - Males & $38(60.3)$ \\
\hline - Females & $25(39.7)$ \\
\hline \multicolumn{2}{|l|}{ Type of seizure $n(\%)$} \\
\hline - Generalized seizures & $39(61.90)$ \\
\hline - Partial/focal seizures & $24(38.10)$ \\
\hline \multicolumn{2}{|c|}{ Overall epilepsy severity using GASE scale* $n(\%)$} \\
\hline - Not at all severe & $11(17.46)$ \\
\hline - A little severe & $38(60.32)$ \\
\hline - Somewhat severe & $13(20.63)$ \\
\hline - Moderately severe & $1(1.59)$ \\
\hline Disease duration** (years), mean (SD) & $4.20(3.63)$ \\
\hline Number of medications, mean (SD) & $1.60(1.01)$ \\
\hline \multicolumn{2}{|l|}{ Number of AED prescribed } \\
\hline - 1 & $47(74.60)$ \\
\hline - 2 & $12(19.05)$ \\
\hline - 3 & $3(4.76)$ \\
\hline - 4 & $1(1.59)$ \\
\hline \multicolumn{2}{|l|}{ Type of AED of interest prescribed, $n(\%)$} \\
\hline - Sodium valproate & $30(47.62)$ \\
\hline - Levetiracetam & $31(49.21)$ \\
\hline - Carbamazepine & $7(11.1)$ \\
\hline \multicolumn{2}{|c|}{ Measured AED concentrations ${ }^{* * *}$ (median $[$ range $], \mu \mathrm{g} / \mathrm{ml}$ ) } \\
\hline - Sodium valproate & $61.64(2.00-136.80)$ \\
\hline - Levetiracetam & $11.61(0.01-42.30)$ \\
\hline - Carbamazepine & $5.91(1.10-12.56)$ \\
\hline \multicolumn{2}{|l|}{ Parent education, n (\%) } \\
\hline - No formal education & $2(3.17)$ \\
\hline - Primary School & $4(6.35)$ \\
\hline - Secondary School & $35(55.56)$ \\
\hline - Bachelor degree & $8(12.70)$ \\
\hline - Master degree or PhD & $14(22.22)$ \\
\hline
\end{tabular}

SD: Standard deviation.

* Among patient's demographic data and disease characteristics, only disease duration was significantly associated with non-adherence to AEDs ( $p$-value $<0.05$ ) 
**(GASE) Scale: Global Assessment of Severity of Epilepsy

*** Patients were considered adherent if their measured AED concentrations were within the calculated $95 \%$ prediction intervals using a pharmacokinetic simulation method.

Table 2. Distribution of the total scores for the MARS questionnaires reported by participating parents and children

\begin{tabular}{lccccc}
\hline \multicolumn{1}{c}{ Measure } & $\mathbf{n}$ & $\begin{array}{c}\text { Total score } \\
\text { mean }\end{array}$ & $\begin{array}{c}\text { Total score } \\
\text { range }\end{array}$ & $\begin{array}{c}\text { Score indicating } \\
\text { non-adherence }\end{array}$ & $\begin{array}{c}\text { Number (\%) of non- } \\
\text { adherent patients }\end{array}$ \\
\hline MARS (Parent) & 63 & 4.65 & $3.5-5$ & $<4.5$ & $13(20.63 \%)$ \\
MARS (Child) & 21 & 4.34 & $1.8-5$ & $<4.5$ & $7(33.33 \%)$ \\
\hline
\end{tabular}

Table 3. Assessment of agreements between the three methods of measuring adherence using Cohen's Kappa analysis

\begin{tabular}{lcc}
\hline Method & Cohen's Kappa Coefficient & p-value \\
\hline AED plasma level vs MARS* (Parent) & 0.187 & 0.133 \\
AED plasma level vs MARS (Child) & 0.131 & 0.585 \\
MARS (Parent) vs MARS (Child) & 0.640 & $0.002 * *$
\end{tabular}

*MARS: Medication Adherence Report Scale

$* *$ Significant at 0.05 level

Table 4 Predictors of non-adherence to AEDs using binary logistic regression

\begin{tabular}{lccccc}
\hline Independent variable & B & SE & Odds ratio & $\mathbf{9 5 \%}$ CI & p-value \\
\hline Disease duration & 0.43 & 0.144 & 1.54 & $1.16-2.04$ & $0.003^{*}$ \\
Parent BMQ-Necessity score & -0.39 & 0.13 & 0.68 & $0.53-0.87$ & $0.002^{*}$ \\
Parent BMQ-Concern score & 0.47 & 0.12 & 1.60 & $1.26-2.04$ & $<0.001^{*}$ \\
\hline $\begin{array}{l}\text { *p-value < 0.05.'Adherence coded 0, non-adherence coded 1. } \\
\text { B, regression coefficient; SE, standard error associated with the coefficient B; CI, confidence interval. }\end{array}$
\end{tabular}




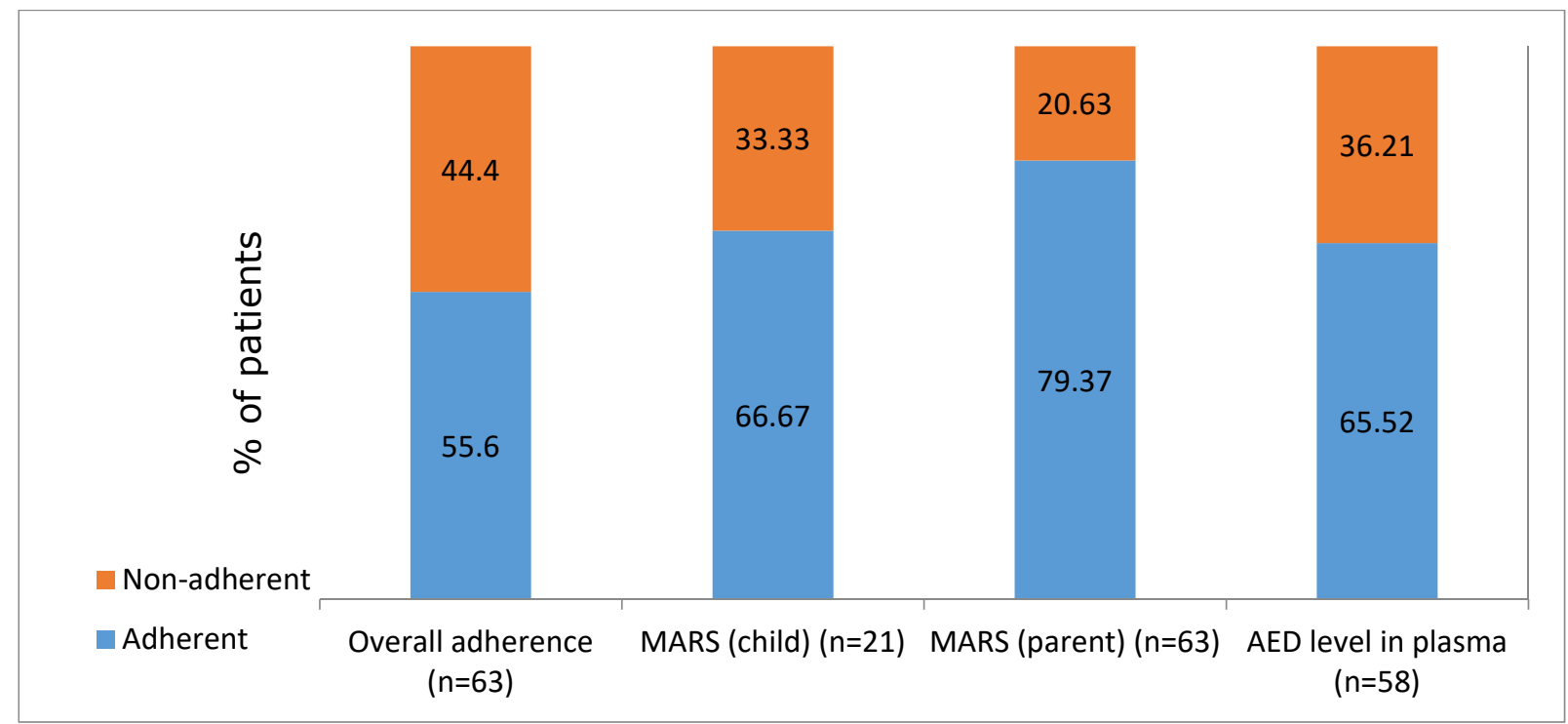

Figure 1.Comparison of different methods of adherence assessment (percentage of adherent patients vs non-adherent patients). 